\title{
B-Line Artifact as a Diagnostic Tool in Various Conditions at the Emergency Department
}

\author{
Kamonwon lenghong ${ }^{1,2}$, Takaaki Suzuki ${ }^{3}$, Ismet Celebi ${ }^{4}$, Vajarabhongsa Bhudhisawasdi ${ }^{1}$, Somsak Tiamkao ${ }^{5}$, \\ Dhanu Gaysonsiri ${ }^{2,6}$, Korakot Apiratwarakul ${ }^{1,2 *}$ \\ ${ }^{1}$ Department of Emergency Medicine, Faculty of Medicine, Khon Kaen University, Khon Kaen, Thailand; ${ }^{2}$ Integrated Emergency \\ Medical Services and Pre-hospital Care Research Group (RG64201), Faculty of Medicine, Khon Kaen University, Khon Kaen, \\ Thailand; ${ }^{3}$ Department of Emergency and Critical Care Medicine, University of Tsukuba Hospital, Tsukuba, Japan; ${ }^{4}$ Department \\ of Paramedic, Gazi University, Ankara, Turkey; ${ }^{5}$ Department of Medicine, Faculty of Medicine, Khon Kaen University, Khon \\ Kaen, Thailand; ${ }^{6}$ Department of Pharmacology, Faculty of Medicine, Khon Kaen University, Khon Kaen, Thailand
}

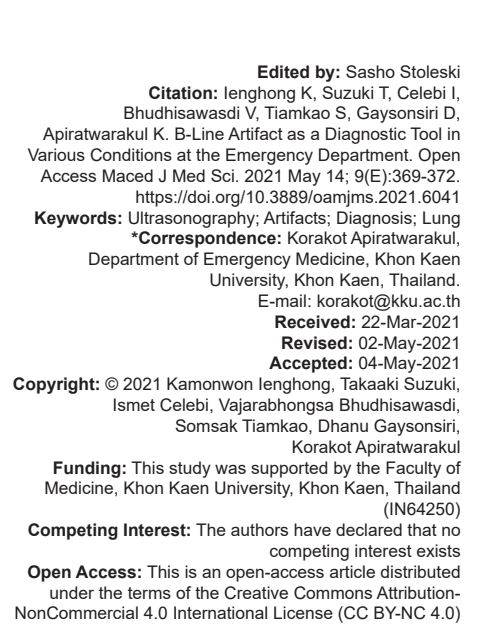

\section{Abstract}

BACKGROUND: B-line artifacts (BLAs) play an important role in identifying lung pathology. They may indicate different diseases. However, the diagnostic study of BLA as applied to emergency patients has not been well studied.

AIM: The aim of this study was to determine the diagnostic accuracy of BLA in various conditions.

METHODS: This was a retrospective observational study of emergency patients who had received lung ultrasound at Srinagarind Hospital's Emergency Department throughout January 2020-December 2020. Ultrasound artifacts were recorded. Ultrasonography findings were correlated with final diagnosis. Sensitivity and specificity were also calculated. RESULTS: A total of 105 patients were evaluated. The most prevalent condition which BLA found in this study was pulmonary edema $(44.12 \%)$ with $88.24 \%$ sensitivity and $46.48 \%$ specificity. BLA also indicated pneumonia with $66.67 \%$ sensitivity and $35.71 \%$ specificity. Diffuse BLA indicated pulmonary edema with $70 \%$ sensitivity and $70.42 \%$ specificity. Focal BLA indicated pneumonia with $28.57 \%$ sensitivity and $76.19 \%$ specificity.

CONCLUSIONS: The sensitivity of BLA for pulmonary edema and pneumonia diagnosis in this study was of moderate to good sensitivity, but low specificity. BLA may become crucial in the diagnosis of lung pathology in the emergency department.

\section{Introduction}

Lung ultrasound (LUS) is an important tool amid the care of patients at the emergency department [1], [2], [3]. Nowadays, LUS is more widely used, especially in critically ill patients [4], [5]. LUS is based on the analysis of ultrasound artifacts. Of the many artifacts, B-line artifact (BLA) is caused by a reverberation phenomenon. Moreover, it is the crucial lung artifact applied in critically ill patients [4]. The characteristic of BLA [6] is as follows: (1) It arises from the pleural line, (2) it is hyperechoic, similar to the pleural line, (3) it is well-defined, similar in appearance to a laser, (4) the sign erases the normal A-lines and extends outward without fading to the bottom of the display screen, and (5) the sign moves with lung sliding. BLA is applied in numerous diseases such as pulmonary edema, congestive heart failure, lung contusions, pneumonia, and acute respiratory distress syndrome [7], [8], [9], [10], [11], [12]. In addition, some literatures showed that BLA has been applied amid connective tissue diseases [13], [14], [15]. While they have been reported on in lung pathology, single focal BLA can also be observed in healthy populations [7].

The previous ultrasound studies [7], [8], [9], [10], [11], [12], [13], [14], [15] have examined selected groups of patients such as those exhibiting acute respiratory distress, dyspnea, or critically ill patients with LUS examination. With the introduction of LUS and training of emergency medicine residents, LUS has been increasingly used in the emergency departments in Thailand. Ultrasound findings through BLA in undifferentiated patients in the emergency department have never been documented. The clinical question addressed in this study was the following: For patients presenting themselves at the emergency department with variable conditions, which disease BLA can be used to confirm a diagnosis accurately? 


\section{Methods}

\section{Study design}

This was a retrospective, single-centered, observational study at a tertiary university hospital in Thailand. Ethical approval was provided by the Khon Kaen University Ethics Committee for Human Research and registered with the Thai Clinical Trials Registry (HE641156).

\section{Sample size}

We included all patients who had received LUS and had had ultrasound video clips and images recorded in the ultrasound machine at the emergency department throughout January 2020-December 2020. Patients with no ultrasound documents were excluded from the study. The sample size for the analysis of the estimated sample size in the diagnostic test was determined. Prevalence was 0.26 [16]. The standard normal value was 1.96 . Power analysis was determined using an alpha of 0.05 . Sens was 0.923 [16] and absolute precision was 0.01 . This resulted in an estimated desired effect sample size of at least 105 subjects.

\section{Study protocol}

The study was performed throughout January 2020-December 2020. We collected data from patients who visited the emergency department. Ultrasound was performed by emergency medicine residents and the attending physician. Ultrasound artifacts including $A$ line and $B$ line were recorded. LUS artifacts were correlated with the final diagnosis made by the treating physicians. This study was conducted with the standard ultrasound machine (Mindray M9) which we used at the emergency department. The images were obtained in B mode. In terms of transducers, the examination of transducer selection depended on the preferences of the physician performing LUS. In this study, we used curvilinear, phased array and linear transducers. The primary outcome of this study aimed to determine the diagnostic accuracy of BLA in various conditions.

\section{Statistical analysis}

Continuous type variables in the data were summarized as mean and standard deviation or median and range as appropriate. Categorical variables were summarized as counts and percentages. Sensitivity and specificity were calculated. All statistical analyses were performed using the software Stata version 10.1 (Stata Corp, College Drive, TX, USA). Statistical significance was defined as a two-sided $p=0.05$ or less.

\section{Results}

Among 105 patients, 52.38\% were female. Median patient age was 64.38 years. The greatest indications of LUS scans were of respiratory symptoms $(50.47 \%)$. BLA was discovered in 68 patients $(64.76 \%)$; diffuse BLA was found in 42 patients $(61.76 \%)$ and focal BLA was discovered in 26 patients (38.23\%). This study included two cardiac arrest patients which one patient receiving diffuse $B L A$ and another patient receiving focal BLA (Table 1).

\section{Table 1: Patients' characteristics}

\begin{tabular}{ll}
\hline Patient characteristics $(\mathrm{n}=105)$ & \\
\hline Sex & $55(52.38)$ \\
$\quad$ Female, $\mathrm{n}(\%)$ & \\
Age & $64.38 \pm 15.44$ \\
$\quad$ Mean \pm SD & \\
LUS artifacts & $37(35.24)$ \\
$\quad$ l line, $\mathrm{n}(\%)$ & $68(64.76)$ \\
B line, $\mathrm{n}(\%)$ & \\
Area of BLAs $(\mathrm{n}=68)$ & $42(61.76)$ \\
$\quad$ Diffuse, $\mathrm{n}(\%)$ & $26(38.24)$ \\
Focal, $\mathrm{n}(\%)$ & \\
\hline BLAs: B-line artifacts, LUS: Lung ultrasound. &
\end{tabular}

$B L A$ revealed various conditions in this study including pulmonary edema $(44.12 \%)$, pneumonia $(20.5 \%)$, septic shock $(10.29 \%)$, lung metastasis (4.41\%), interstitial lung disease (ILD) (systemic sclerosis, systematic lupus erythematous, and PCP infection) (2.94\%), and pulmonary embolism (2.94\%).

Besides that, BLA specified pulmonary edema with $88.24 \%$ sensitivity and $46.48 \%$ specificity. BLA indicated pneumonia with $66.67 \%$ sensitivity and $35.71 \%$ specificity. Diffuse BLA specified pulmonary edema with $70 \%$ sensitivity and $70.42 \%$ specificity. Focal BLA indicated pneumonia with $28.57 \%$ sensitivity and $76.19 \%$ specificity (Table 2 ).

Table 2: Sensitivity and specificity of BLA categorized by diseases

\begin{tabular}{lll}
\hline BLA observed in final diagnosis & Sensitivity (\%) & Specificity (\%) \\
\hline Pulmonary edema $(n=34)$ & 88.24 & 46.48 \\
Pneumonia $(n=21)$ & 66.67 & 35.71 \\
Septic shock $(n=3)$ & 100 & 62.24 \\
Lung metastasis $(n=3)$ & 100 & 36.27 \\
ILD $(n=5)$ & 40 & 66 \\
Pulmonary embolism ( $n=2)$ & 100 & 64.28 \\
\hline BLA: B-line artifact, ILD: Interstitial lung disease. & &
\end{tabular}

\section{Discussion}

The present study described 105 patients who underwent LUS at the emergency department. Our study found BLA in $64.76 \%$ of patients. Hence, BLA indicated pulmonary edema with $88.24 \%$ sensitivity in consistence with other studies [5]; however, rather low specificity was established $(46.48 \%)$. The study of Lichtenstein and Mezière [5] revealed the discovery of multiple anterior diffuse $B$ lines, with lung sliding indicating pulmonary edema with $97 \%$ sensitivity and $95 \%$ specificity. From 
a meta-analysis study [17], diffuse BLA for diagnosis of pulmonary edema was more accurate than initial clinical work-up, chest X-ray, and natriuretic peptides [18].

In terms of pneumonia, we discovered diffuse BLA in $>50 \%$ of pneumonia cases. In accordance with Patel et al. [16], they found bilateral BLA (more than two $B$ lines) in two or more zones out of a sixzone examination with or without pleural line and subpleural abnormalities which were suggestive of this diagnosis. Our study showed $66.67 \%$ sensitivity and $35.71 \%$ specificity which was a lower rate than in other studies [5]. These results displayed anterior diffuse $B$ lines with abolished lung sliding which indicated pneumonia with $89 \%$ sensitivity and $94 \%$ specificity.

In terms of septic shock, most physicians in this study employed LUS to assess fluid responsiveness. We established that most septic shock patients in this study had fluid overload from LUS that showed diffuse BLA. However, due to this method of data collection, we are unable to establish whether the volume of fluid resuscitation was associated with BLA in LUS or not.

ILD is a major pulmonary manifestation of connectivetissuedisease. Ourstudy demonstrated 100\% sensitivity of BLA to detect ILD from systemic sclerosis, systematic lupus erythematous, and PCP infection. In accordance with a study by Barskova et al. [4], they reported that LUS diagnostic sensitivity and specificity were $100 \%$ and $55 \%$, respectively. Moreover, negative predictive value and positive predictive value were $100 \%$ and $78 \%$. Numerous B line in LUS was associated with high-resolution computed tomography findings in connective tissue disease.

The strengths of this study were as follows: (1) We identified the sensitivity and specificity of BLA for diagnosis in various conditions in Thai emergency patients and (2) the findings from this study demonstrated the impact of using POCUS at the emergency department. Limitations of the study were as follows [19], [20], [21], [22], [23]: (1) It could not assess certain conditions at the emergency department such as fluid responsiveness which was mentioned above, and (2) in this study, the data collected from various types of transducer may have influenced BLA image quality.

\section{Conclusions}

BLA was applied to observe pulmonary edema and pneumonia with moderate to good sensitivity in emergency patients. BLA may become crucial in directing the diagnostic process. Further research is warranted to clarify technical adjustments as well as different transducers and machine factors which influence BLA visualization.

\section{Acknowledgments}

The authors would like to thank all participants in this study as well as Mr. Ross Lacey for acting as an English The present study received fund from Faculty of Medicine, Khon Kaen University, Khon Kaen, Thailand (IN64250).

\section{References}

1. Koenig SJ, Narasimhan M, Mayo PH. Thoracic ultrasonography for the pulmonary specialist. Chest. 2011;140(5):1332-41. https://doi.org/10.1378/chest.11-0348 PMid:22045878

2. Hew M, Heinze S. Chest ultrasound in practice: A review of utility in the clinical setting. Intern Med J. 2012;42(8):856-65. PMid:22530570

3. Volpicelli G, Elbarbary M, Blaivas M, Lichtenstein DA Mathis G, Kirkpatrick AW, et al. International evidence-based recommendations for point-of-care lung ultrasound. Intensive Care Med. 2012;38(4):577-91. https://doi.org/10.1007/ s00134-012-2513-4

PMid:22392031

4. Mojoli F, Bouhemad B, Mongodi S, Lichtenstein D. Lung ultrasound for critically III patients. Am J Respir Crit Care Med. 2019;199(6):701-14. https://doi.org/10.1164/ rccm.201802-0236ci

PMid:30372119

5. Lichtenstein DA, Mezière GA. Relevance of lung ultrasound in the diagnosis of acute respiratory failure: The BLUE protocol. Chest. 2008;134(1):117-25. https://doi.org/10.1378/ chest.07-2800

PMid:18403664

6. Lichtenstein DA. Lung ultrasound in the critically III. J Med Ultrasound. 2009;17(3):125-42.

PMid:24401163

7. Facchini C, Malfatto G, Giglio A, Facchini M, Parati G, Branzi G. Lung ultrasound and transthoracic impedance for noninvasive evaluation of pulmonary congestion in heart failure. J Cardiovasc Med (Hagerstown). 2015;17(7):510-7. https://doi.org/10.2459/ jcm.0000000000000226

PMid:25575275

8. Miglioranza MH, Gargani L, Sant'Anna RT, Rover MM, Martins VM, Mantovani A, et al. Lung ultrasound for the evaluation of pulmonary congestion in outpatients: A comparison with clinical assessment, natriuretic peptides, and echocardiography. JACC Cardiovasc Imaging. 2013;6(11):114151. https://doi.org/10.1016/j.jcmg.2013.08.004

PMid:24094830

9. Weber CK, Miglioranza MH, Moraes MA, Sant'anna RT, Rover MM, Kalil RA, et al. The five-point Likert scale for dyspnea can properly assess the degree of pulmonary congestion and predict adverse events in heart failure outpatients. Clinics (Sao Paulo). 2014;69(5):341-6. https://doi.org/10.6061/ clinics/2014(05)08

PMid:24838900

10. Frassi F, Pingitore A, Cialoni D, Picano E. Chest sonography detects lung water accumulation in healthy elite apnea divers J Am Soc Echocardiogr. 2008;21(10):1150-5. https://doi. 
org/10.1016/j.echo.2008.08.001

PMid:18926391

11. Edsell ME, Wimalasena $\mathrm{YH}$, Malein WL, Ashdown KM Gallagher CA, Imray $\mathrm{CH}$, et al. High-intensity intermittent exercise increases pulmonary interstitial edema at altitude but not at simulated altitude. Wilderness Environ Med. 2014;25(4):409-15. https://doi.org/10.1016/j.wem.2014.06.016 PMid:25443761

12. Volpicelli G, Skurzak S, Boero E, Carpinteri G, Tengattini M, Stefanone $\mathrm{V}$, et al. Lung ultrasound predicts well extravascular lung water but is of limited usefulness in the prediction of wedge pressure. Anesthesiology. 2014;121(2):320-7. https://doi. org/10.1097/aln.0000000000000300

PMid:24821071

13. Wang $Y$, Gargani L, Barskova T, Furst DE, Cerinic MM Usefulness of lung ultrasound B-lines in connective tissue disease-associated interstitial lung disease: A literature review. Arthritis Res Ther. 2017;19(1):206. https://doi.org/10.1186/ s13075-017-1409-7

PMid:28923086

14. Barskova T, Gargani L, Guiducci S, Randone SB, Bruni C, Carnesecchi $G$, et al. Lung ultrasound for the screening of interstitial lung disease in very early systemic sclerosis. Ann Rheum Dis. 2013;72(3):390-5. https://doi.org/10.1136/ annrheumdis-2011-201072

PMid:22589373

15. Gargani L, Bruni C, Romei C, Frumento P, Moreo A, Agoston G, et al. Prognostic value of lung ultrasound B-lines in systemic sclerosis. Chest. 2020;158(4):1515-25. https://doi.org/10.1016/j. chest.2020.03.075

PMid:32360727

16. Patel CJ, Bhatt HB, Parikh SN, Jhaveri BN, Puranik JH. Bedside lung ultrasound in emergency protocol as a diagnostic tool in patients of acute respiratory distress presenting to emergency department. J Emerg Trauma Shock. 2018;11(2):125-9. https:// doi.org/10.4103/jets.jets_21_17

PMid:29937643

17. Al Deeb M, Barbic S, Featherstone R, Dankoff J, Barbic D. Pointof-care ultrasonography for the diagnosis of acute cardiogenic pulmonary edema in patients presenting with acute dyspnea: A systematic review and meta-analysis. Acad Emerg Med. 2014;21(8):843-52. https://doi.org/10.1111/acem.12435 PMid:25176151

18. Pivetta E, Goffi A, Lupia E, Tizzani M, Porrino G, Ferreri E, et al. Lung ultrasound-implemented diagnosis of acute decompensated heart failure in the ED: A SIMEU multicenter study. Chest. 2015;148(1):202-10. https://doi.org/10.1093/ eurheartj/eht308.1723 PMid:25654562

19. Apiratwarakul $K$, lenghong $K$, Gaysonsiri $D$, Mitsungnern $T$, Buranasakda M, Bhudhisawasdi V. The effectiveness of oxygen-powered inhalation devices in pre-hospital care. J Med Assoc Thai. 2020;103 Suppl 6:58-60.

20. lenghong K, Towsakul N, Bhudhisawasdi V, Srimookda N, Ratanachotmanee N, Phungoen P. POCUS findings in critically III patients in emergency department. J Med Assoc Thai. 2021;104 Suppl 1:S54-8. https://doi.org/10.35755/jmedassocthai.2021. s01.12228

21. lenghong $\mathrm{K}$, Kleebbuakwan $\mathrm{K}$, Apiratwarakul K, Phungoen $\mathrm{P}$, Gaysonsiri D, Bhudhisawasdi V. Comparison of cleaning methods for ultrasound probes at an emergency department in a resource-limited country. J Med Assoc Thai. 2020;103 Suppl 6:67-71.

22. Apiratwarakul $K$, Songserm $W$, lenghong $K$, Phungoen $P$, Gaysonsiri D, Bhudhisawasdi V. The role of mechanical cardiopulmonary resuscitation devices in emergency medical services. J Med Assoc Thai. 2020;103 Suppl 6:98-101.

23. Apiratwarakul K, lenghong K, Bhudhisawasdi V, Gaysonsiri D, Tiamkao S. Response times of motorcycle ambulances during the COVID-19 pandemic. Open Access Maced J Med Sci. 2020;8(T1):526-9. https://doi.org/10.3889/oamjms.2020.5527 